Article

\title{
A Method for Balancing a Single-Phase Loaded Three-Phase Induction Generator
}

\author{
Yaw-Juen Wang and Ming-Hsueh Lee *
}

Graduate School of Engineering Science and Technology, National Yunlin University of Science \& Technology, 123 University Road, Section 3, Douliou, Yunlin 64002, Taiwan;

E-Mail: wangyj@yuntech.edu.tw

* Author to whom correspondence should be addressed; E-Mail: g9310807@yuntech.edu.tw; Tel.: +886-5534-2601; Fax: +886-5531-2065.

Received: 18 May 2012; in revised form: 23 August 2012 / Accepted: 10 September 2012 /

Published: 13 September 2012

\begin{abstract}
When a three-phase induction generator (IG) supplies unbalanced loads, its terminal voltages and line currents are also unbalanced, which may cause the IG to overheat and need to be derated. A single-phase loaded self-excited induction generator (SEIG) works under most unfavorable load unbalance conditions. This paper proposes a three-capacitor circuit scheme and a method to find the values of the self-excitation capacitors that allow the SEIG to be balanced. The SEIG is modeled by a two-port network equivalent circuit that resolves the SEIG into its positive- and negative-sequence circuits associated with the self-excitation capacitors and the load. The network can then be analyzed by common AC circuit analysis techniques. Successful results for balancing the SEIG supplying a single-phase load have been achieved by properly choosing the values of the excitation capacitors. The proposed method has also been validated by experiments on a $0.37 \mathrm{~kW}$ SEIG.
\end{abstract}

Keywords: induction generator; unbalance; single-phase load

\section{Symbols and Acronyms}

$C_{1}, C_{2}, C_{3}$ excitation capacitances

$F \quad$ per-unit frequency

$I_{0}, I_{p}, I_{n} \quad$ zero-, positive- and negative-sequence currents

$I_{a}, I_{b}, I_{c} \quad$ three-phase line currents

$I_{m} \quad$ magnetizing current

$I_{r} \quad$ positive-sequence component of the rotor current 
$I_{s p}, I_{s n} \quad$ positive- and negative-sequence stator currents

$R_{s}, R_{r} \quad$ stator and rotor resistances

$v \quad$ per-unit speed

$V_{a}, V_{b}, V_{c}$ three-phase phase voltages

$V_{\mathrm{g}} \quad$ air-gap voltage

$V_{p}, V_{n} \quad$ positive- and negative-sequence voltages

$X_{c r} \quad$ critical magnetizing reactance

$X_{m} \quad$ magnetizing reactance

$X_{m n} \quad$ negative-sequence magnetizing reactance

$X_{s}, X_{r} \quad$ stator and rotor reactances

$y_{1}, y_{2}, y_{3} \quad$ load admittances

$y_{\alpha}, y_{\beta}, y_{\mathrm{d}}$ functions of $y_{1}, y_{2}$ and $y_{3}$ defined by Equation (6)

$Y_{L} \quad$ admittance looking to the right of terminals $h$ and $k$ in Figure 3

$Y_{n t h} \quad$ admittance to the right of terminals $l$ and $m$ in Figure 3

$Y_{r} \quad$ rotor admittance

$Y_{\mathrm{s}} \quad$ stator admittance

$Y_{T} \quad$ function of $Y_{r}, Y_{s}$ and $Y_{L}$ defined by Equation (15)

$\mathrm{Z}_{L} \quad$ single-phase load impedance

$\boldsymbol{V}, \boldsymbol{I} \quad$ voltage and current vectors

$\boldsymbol{V}_{\mathrm{s}}, \boldsymbol{I}_{\mathrm{s}} \quad$ voltage and current symmetrical component vectors

$\boldsymbol{Y}$ nodal admittance matrix

$\boldsymbol{Y}_{\mathrm{S}} \quad$ similarity transformation of admittance matrix $\boldsymbol{Y}$

$\alpha \quad$ firing angle

$\tau \quad$ voltage unbalance factor

CUF current unbalance factor

IG induction generator

IM induction motor

SEIG self-excited induction generator

SVC static var compensator

TCR thyristor-controlled reactor

VUF voltage unbalance factor

\section{Introduction}

Induction generators (IGs) are receiving more attention than in the past because of their low prices, simple construction, little maintenance, ruggedness and the fact they do not need dc sources and brushes, which make them particularly suitable as an energy conversion device for renewable energy sources such as wind, biogas and micro-hydro. In many developing countries where a significant percentage of the population and areas still suffer from electricity shortages, employing IGs for 
generating electricity from renewable energy has become an important means of rural electrification and reduction of greenhouse gases emissions [1].

IGs can be operated in stand-alone or grid-interactive mode. In grid-interactive mode, an IG is directly connected to the grid that imposes its voltage and frequency and supplies the required reactive power. The analysis of a grid-connected IG does not differ much from that of an induction motor (IM). On the other hand, in stand-alone mode, self-excitation capacitors are needed to supply reactive power to the generator and load, and the generated voltage and frequency depend on the rotational speed, the load impedance and the values of excitation capacitors. Hence, the analysis of a self-excited induction generator (SEIG) is more complicated.

People living off the grid in remote islands, rural or mountain areas usually have single-phase type loads. Hence, a single-phase SEIG is an ideal option for electric power supply. While high capacity single-phase IMs up to $10 \mathrm{hp}$ are available in the market, three-phase IMs above $5 \mathrm{hp}(3.7 \mathrm{~kW})$ are cheaper than single-phase IMs of the same ratings [2]. The use of a three-phase IM operated as an SEIG to generate single-phase electricity has been employed in many places for economic reasons. However, a three-phase SEIG operated as a single-phase generator is working under a very unbalanced condition, necessitating it to be derated to avoid overheating.

In the literature, studies on the three-phase SEIG feeding single-phase loads can roughly be categorized into two groups. The first group endeavors to minimize the voltage regulation and at the same time maximize the single-phase power output of the generator by properly choosing the values of the self-excitation capacitors, considering possible changes in the rotational speed of the prime mover. To mention some examples, Wang and Cheng [3] determined the minimum and maximum values of a single excitation capacitor required by a three-phase SEIG supplying a single-phase resistive load using the eigenvalue sensitivity method. Fukami et al. [4] proposed and analyzed a self-regulated three-phase SEIG working as a single-phase generator that consisted of two equal capacitors connected in series and another capacitor in parallel with the load resistor, and provided improved performance of voltage regulation. This generator scheme was further optimized by Mahato et al. [5] using the sequential unconstrained minimization technique to obtain a maximum power output for both capacitive and inductive single-phase loads.

The second group stresses the need for mitigating the voltage and current unbalance of a three-phase SEIG working as a single-phase generator. Al-Bahrani and Malik [6] analyzed the performance of a three-phase SEIG composed of a single capacitor in parallel with a single-phase load, i.e., the single-phasing mode. Chan [7] developed a method based on symmetrical components for analyzing a three-phase SEIG supplying a single-phase load. Chan's method was applicable to the single-phasing mode as well as the Steinmetz connection. Chan and Lai $[8,9]$ also developed a method to determine the minimum capacitance for voltage building-up of a three-phase SEIG with Steinmetz connection. It is noted that [6-9] only give methods for analyzing a three-phase SEIG feeding a single-phase load and do not report any method for balancing it. Chan and Lai [10] proposed a circuit scheme to balance the SEIG. In addition to the load resistance and excitation capacitance in the Steinmetz connection, they added an auxiliary load resistance and an auxiliary excitation capacitance. They used a phasor diagram to explain how to find the appropriate values of the auxiliary resistance and capacitance that allow a perfectly balanced three-phase voltage and current to be obtained. Alolah and Alkanhal [11] proposed another scheme that consisted of two excitation capacitors, the values of 
which were determined using a sequential genetic and gradient optimization method. The voltage unbalance factor of the SEIG using the capacitance values found by that proposed algorithm was reported to range from $2 \%$ to $7 \%$, showing a system that is not perfectly balanced.

In fact, achieving balanced loading on a three-phase generator provides benefits to the generator's performance which include: (1) making full use of the generator rating; (2) eliminating the pulsating torque caused by the negative-sequence current and hence reducing shaft mechanical vibration; and (3) eliminating voltage unbalance at the generator terminals caused by unbalanced load currents, which also improves voltage regulation of the generator.

In this paper, the authors propose a three-capacitor scheme that is able to perfectly balance a three-phase SEIG supplying a single-phase load. The three capacitors include a fixed capacitor that supplies the required reactive power to the system, and two variable capacitors that play the role of balancer. The two variable capacitors can be realized by two static var compensators (SVCs), a parallel combination of a fixed capacitor and a thyristor-controlled reactor (TCR). The three-capacitor scheme is represented by a two-port network IG model developed by Wang and Huang [12] for analysis. A solution method based on this two-port network model is also proposed for determining the values of the three capacitors. The circuit scheme and the method for finding capacitances to balance the SEIG are validated by comparing results obtained by the two-port network SEIG model and by experiments on a $0.375 \mathrm{~kW}$ induction generator.

\section{Generator and Load Modeling}

The two-port network model for a three-phase SEIG supplying unbalanced load developed in [12] is briefly described here and is used to analyze the proposed generator scheme. In addition, the voltage unbalance factor expressed as a function of the two-port network model's parameters is also derived.

\subsection{Generator Scheme}

Figure 1 shows the proposed three-capacitor scheme, which is composed of a $\Delta$-connected three-phase induction machine and three capacitors $C_{1}, C_{2}$ and $C_{3}$ across each terminal pair. The single-phase load impedance $Z_{L}$ is connected across terminals $a$ and $b$. Capacitors $C_{2}$ and $C_{3}$ are variable according to the generator rotational speed and the load impedance, while $C_{1}$ is fixed and serves to supply additional reactive power to the machine and load. We note that the scheme shown in Figure 1 is indeed an IG supplying a $\Delta$-connected unbalanced load composed of three admittances $y_{1}, y_{2}$ and $y_{3}$.

Figure 1. Three-capacitor scheme of SEIG feeding a single-phase load.

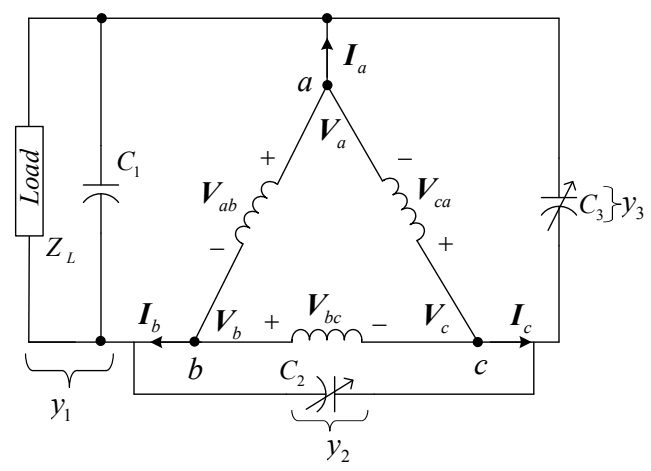




\subsection{Modelling of an Unbalanced Three-Phase Load}

Unbalanced loads can be combined and modeled as a $\Delta$-connected three-phase load as shown in Figure 2a, where $y_{1}, y_{2}$ and $y_{3}$ are load admittances. Mixture of Y-connected and single-phase loads can also be converted to this model by appropriate transformation formulae. In Figure $2 \mathrm{a}, V_{a}, V_{b}$ and $V_{c}$ refer to the phase voltages at the load terminals, and $I_{a}, I_{b}$ and $I_{c}$ to the line currents flowing into the load.

The relation between the three-phase voltages and currents can be written as:

$$
\boldsymbol{I}=\left[\begin{array}{c}
I_{a} \\
I_{b} \\
I_{c}
\end{array}\right]=\left[\begin{array}{ccc}
y_{3}+y_{1} & -y_{1} & -y_{3} \\
-y_{1} & y_{1}+y_{2} & -y_{2} \\
-y_{3} & -y_{2} & y_{2}+y_{3}
\end{array}\right]\left[\begin{array}{c}
V_{a} \\
V_{b} \\
V_{c}
\end{array}\right]=\boldsymbol{Y} \boldsymbol{V}
$$

where $\boldsymbol{I}$ and $\boldsymbol{V}$ are current and voltage vectors, respectively; and $\boldsymbol{Y}$ the nodal admittance matrix. Let the corresponding symmetrical voltage and current component vectors be $\boldsymbol{V}_{\mathrm{s}}$ and $\boldsymbol{I}_{\mathrm{s}}$, respectively. $\boldsymbol{V}_{\mathrm{s}}$ and $\boldsymbol{I}_{\mathrm{s}}$ can be expressed as:

$$
\boldsymbol{V}_{s}=\left[\begin{array}{lll}
V_{0} & V_{p} & V_{n}
\end{array}\right]^{T}, \boldsymbol{I}_{s}=\left[\begin{array}{lll}
I_{0} & I_{p} & I_{n}
\end{array}\right]^{T}
$$

The subscripts $0, p$ and $n$ refer to zero-, positive- and negative-sequence components, respectively. From Equation (1) and the symmetrical component equation for currents, we can write:

$$
\boldsymbol{I}_{s}=\boldsymbol{A}^{-1} \boldsymbol{I}=\boldsymbol{A}^{-1} \boldsymbol{Y} \boldsymbol{V}=\boldsymbol{A}^{-1} \boldsymbol{Y} \boldsymbol{A} \boldsymbol{V}_{s}=\boldsymbol{Y}_{s} \boldsymbol{V}_{s}
$$

where:

$$
\boldsymbol{Y}_{s}=\boldsymbol{A}^{-1} \boldsymbol{Y} \boldsymbol{A}, \boldsymbol{A}=\left[\begin{array}{ccc}
1 & 1 & 1 \\
1 & a^{2} & a \\
1 & a & a^{2}
\end{array}\right], a=1 \angle 120^{\circ}
$$

In Equation (4), $\boldsymbol{Y}_{\mathrm{S}}$ is the similarity transformation of admittance matrix $\boldsymbol{Y}$. Equation (4) in a more detailed form can be given by:

$$
\boldsymbol{Y}_{s}=\left[\begin{array}{ccc}
0 & 0 & 0 \\
0 & y_{d} & y_{\alpha} \\
0 & y_{\beta} & y_{d}
\end{array}\right]
$$

where:

$$
y_{\alpha}=-\left(a y_{1}+y_{2}+a^{2} y_{3}\right), y_{\beta}=-\left(a^{2} y_{1}+y_{2}+a y_{3}\right), y_{d}=y_{1}+y_{2}+y_{3}
$$

Hence, Equation (3) can be rewritten as:

$$
\left[\begin{array}{l}
I_{0} \\
I_{p} \\
I_{n}
\end{array}\right]=\left[\begin{array}{ccc}
0 & 0 & 0 \\
0 & y_{d} & y_{\alpha} \\
0 & y_{\beta} & y_{d}
\end{array}\right]\left[\begin{array}{l}
V_{0} \\
V_{p} \\
V_{n}
\end{array}\right]
$$

Since the zero-sequence current $I_{0}=0$, Equation (7) can be written in a more compact form as:

$$
\left[\begin{array}{c}
I_{p} \\
I_{n}
\end{array}\right]=\left[\begin{array}{ll}
y_{d} & y_{\alpha} \\
y_{\beta} & y_{d}
\end{array}\right]\left[\begin{array}{l}
V_{p} \\
V_{n}
\end{array}\right]
$$


Equation (8) implies a two-port network as shown in Figure 2b.

Figure 2. A delta-connected three-phase unbalanced load. (a) Circuit showing the nodal voltages and line currents; (b) The corresponding two-port network equivalent circuit.

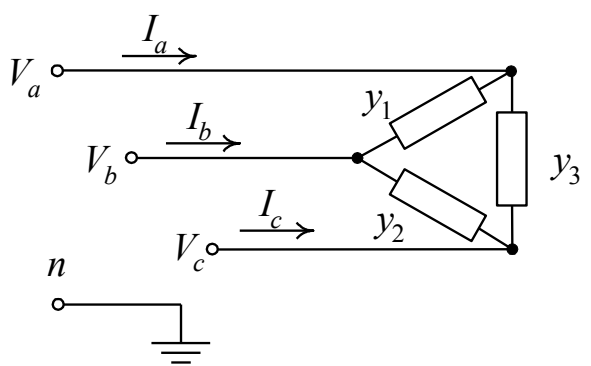

(a)

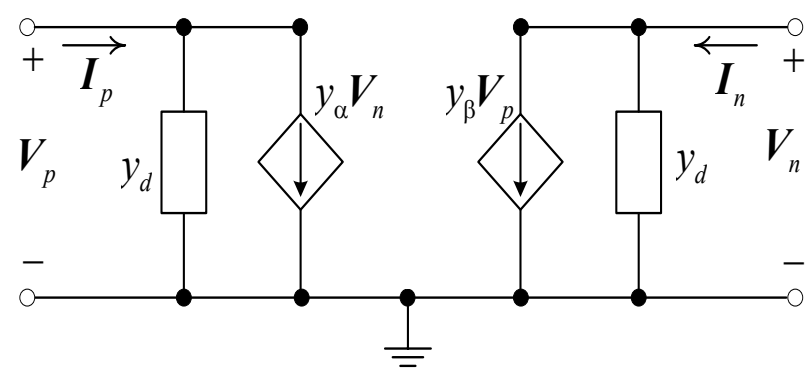

(b)

\subsection{Combination of Load and Generator Models}

The load model shown in Figure $2 \mathrm{~b}$ and the generator positive- and negative-sequence equivalent circuits can be combined to represent the SEIG with unbalanced loads. It is noted that voltages $V_{p}$ and $V_{n}$ and admittances $y_{\alpha}, y_{\beta}$ and $y_{d}$ shown in Figure $2 \mathrm{~b}$ need to be corrected by the per-unit frequency $F$. The complete sequence network of the SEIG with unbalanced loads is depicted in Figure 3, in which the left part is the positive-sequence network while the right part is the negative-sequence network. The two parts interact with each other through two dependent current sources. The effects of load unbalance are clearly expressed by parameters $y_{\alpha}, y_{\beta}$ and $y_{d}$. It is noted again that $y_{\alpha}, y_{\beta}$ and $y_{d}$ are functions of $F$. When three-phase loads are balanced, i.e., $y_{1}=y_{2}=y_{3}$, both $y_{\alpha}$ and $y_{\beta}$ are equal to zero, implying no interaction between the positive- and the negative-sequence networks. The two-port equivalent circuit in Figure 3 fully represents the SEIG supplying unbalanced loads. A single-phase load is a particular case, and also the worst case, of unbalanced loads. In Figure 3, $Y_{r}$ refers to the rotor admittance, $Y_{\mathrm{s}}$ to the stator admittance, and $Y_{L}$ to the admittance looking to the right of terminals $h$ and $k$. $Y_{n t h}$ is the admittance to the right of terminals $l$ and $m$. Calculation of $Y_{n t h}$ is made easier if $j X_{m n}$ is removed. This is a good approximation since the parallel branch $\left|\left(R_{r} / F+v\right)+j X_{r}\right|$ is much smaller than $\left|j X_{m n}\right|$, which gives:

$$
Y_{n t h}=y_{d}+\left[\frac{R_{s}}{F}+\frac{R_{r}}{F+v}+j\left(X_{s}+X_{r}\right)\right]^{-1}
$$

The circuit shown in Figure 3 allows the positive-sequence stator current $I_{s p}$ to be written as:

$$
I_{s p}=Y_{L} \cdot\left(\frac{V_{p}}{F}\right)=y_{d} \cdot \frac{V_{p}}{F}+y_{\alpha} \cdot \frac{V_{n}}{F}
$$

in which $V_{\mathrm{n}} / F$ can be given by:

$$
\frac{V_{n}}{F}=-\frac{1}{Y_{n t h}} \cdot y_{\beta} \cdot \frac{V_{p}}{F}
$$

Combining Equations $(10,11)$ allows $I_{s p}$ to be written as: 


$$
I_{s p}=\left(y_{d}-\frac{y_{\alpha} y_{\beta}}{Y_{n t h}}\right) \cdot \frac{V_{p}}{F}=Y_{L} \cdot \frac{V_{p}}{F}
$$

which implies:

$$
Y_{L}=y_{d}-\frac{y_{\alpha} y_{\beta}}{Y_{n t h}}
$$

Figure 3. Two-port network model of a three-phase SEIG with unbalanced load.

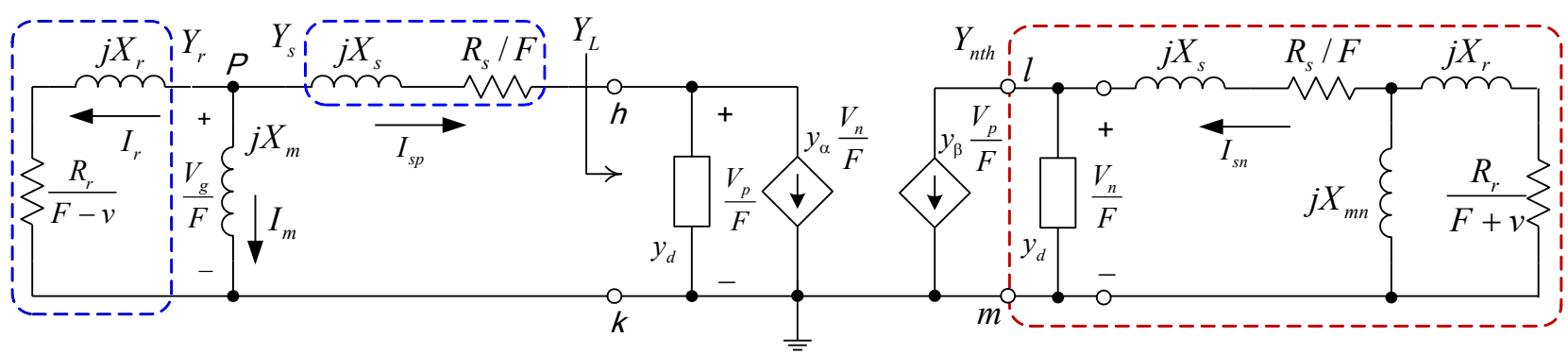

\subsection{Analysis of the Sequence Networks}

Analysis of the circuit of Figure 3 aims at finding the voltages and currents of the SEIG so that its performance under a specified operation condition can be predicted. In this paper, a two-step method is used to find the per-unit frequency $F$ and the magnetizing reactance $X_{m}$ in sequence. In Figure 3, we specify the air-gap voltage $\boldsymbol{V}_{\mathrm{g}}$ to be the reference phasor. That is, $\boldsymbol{V}_{g}=V_{g} \angle 0^{\circ}$. The magnetizing current $I_{m}$ must be a pure imaginary quantity since the magnetizing reactance $j X_{m}$, which is a saturable reactance, is a pure imaginary quantity. The sum of the currents leaving node $P$ equals zero, which means that $\left(I_{r}+I_{s p}\right)=-I_{m}$ is also an imaginary number. The sum $\left(I_{r}+I_{s p}\right)$ can be given by:

$$
I_{r}+I_{s p}=\frac{V_{g}}{F} Y_{r}+\frac{\left(V_{g} / F\right)}{Y_{L}^{-1}+Y_{s}^{-1}}=\frac{V_{g}}{F} \cdot\left(Y_{r}+\frac{Y_{L} Y_{s}}{Y_{L}+Y_{s}}\right)
$$

Since $V_{\mathrm{g}}$ is a real quantity and $\left(I_{r}+I_{s p}\right)$ an imaginary quantity, the real part of the admittance $Y_{r}+\frac{Y_{L} Y_{s}}{Y_{L}+Y_{s}}$ must be zero. If we define:

$$
Y_{T}(F)=Y_{r}+Y_{L} Y_{s} /\left(Y_{L}+Y_{s}\right)
$$

then the following relation holds:

$$
\operatorname{Re}\left[Y_{T}(F)\right]=\operatorname{Re}\left[Y_{r}+Y_{L} Y_{s} /\left(Y_{L}+Y_{s}\right)\right]=0
$$

Since $Y_{T}$ is a function of $Y_{r}, Y_{s}$ and $Y_{L}$, it is also a function of the per-unit frequency $F$. Equation (16) has only one unknown $F$, and can be solved for $F$ using a numerical method such as the secant method or the false position method [13]. Determination of $F$ does not need to know the value of $X_{m}$, largely simplifying the analysis. Once $F$ is obtained by solving Equation (16), $Y_{T}$ can then be found. Note that $Y_{T}$ is indeed the total admittance in parallel with $j X_{m}$. The following relation holds:

$$
I_{m}\left(j X_{m}+1 / Y_{T}\right)=0
$$


Hence, we have:

$$
j X_{m}=-1 / Y_{T}
$$

After the per-unit frequency $F$ is obtained, the air-gap voltage $V_{\mathrm{g}}$ is to be determined. Figure 4 shows that the working point is at the intersection of the magnetizing curve and a load line defined by the equation:

$$
V_{g} / F=j X_{m} \cdot I_{m}=-I_{m} / Y_{T}
$$

Figure 4. Determination of the air-gap voltage $V_{\mathrm{g}}$ and the maximum magnetizing reactance $X_{c r}$.

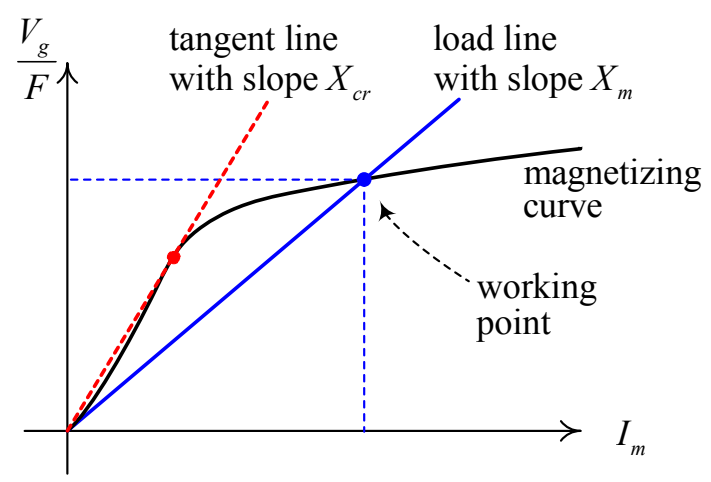

The expression of $Y_{T}$ has been given in Equation (15). An iterative procedure can be taken to find the working point. The iterative procedure starts at an initial value of $V_{\mathrm{g}}$ or $I_{m}$, and finally converges at the intersection point. It is noted that the magnetizing curve must be obtained from the experiment. As shown in Equation (19), the slope of the load line is equal to $X_{m}$, which is a function of the rotational speed, the load impedance $Z_{L}$ and the excitation capacitances $C_{1}, C_{2}$ and $C_{3}$. When the load line is just tangent to the magnetizing curve as shown by the (red) tangent line in Figure $4, X_{m}$ attains a critical value $X_{c r}$ over which the generator is unable to build voltage. Hence, comparing the obtained $X_{m}$ with $X_{c r}$ is a useful criterion to judge if the generator can build voltage.

When $F, V_{\mathrm{g}}$ and $I_{m}$ are known, all the voltages and currents in Figure 3 are easily obtainable. The voltages and currents shown in Figure 3 are positive- and negative-sequence components that need to be converted into the phase domain to find the corresponding three-phase quantities. An important quantity is the voltage unbalance factor $\tau$, defined as the magnitude of the ratio of the negative- to positive-sequence voltage. From (11), the voltage unbalance factor $\tau$ can be expressed as a function of $y_{\beta}$ and $Y_{n t h}$ :

$$
\tau=V U F=\left|\frac{V_{n}}{V_{p}}\right|=\left|\frac{y_{\beta}}{Y_{n t h}}\right|
$$

\section{The Solution Method}

The analysis in Section 2 reveals that the voltage unbalance factor $\tau$ of the SEIG is determined by the ratio of $y_{\beta}$ to $Y_{n t h}$. When the SEIG is working at a balanced condition, the voltage unbalance factor $\tau$ must be equal to zero, which means that the numerator of Equation (20) is zero. Namely, the real and imaginary parts of admittance $y_{\beta}$ must both be zero. These two conditions combined with the relation 
for the per unit frequency $F$ described by Equation (16) allow the following three simultaneous nonlinear equations to be written:

$$
\begin{aligned}
& \operatorname{Re}\left[y_{\beta}(F)\right]=\operatorname{Re}\left[-\left(a^{2} \cdot y_{1}+y_{2}+a \cdot y_{3}\right)\right]=0 \\
& \operatorname{Im}\left[y_{\beta}(F)\right]=\operatorname{Im}\left[-\left(a^{2} \cdot y_{1}+y_{2}+a \cdot y_{3}\right)\right]=0
\end{aligned}
$$

Given that $C_{1}$ and $Z_{L}$ are known, the values of $F, C_{2}$ and $C_{3}$ can be found by solving Equations $(16,21,22)$ using the Newton-Raphson method if appropriate initial guess values are provided. The obtained values of $C_{2}$ and $C_{3}$ allow the SEIG to work at a balanced condition. In comparison with the Hooke and Jeeves search technique used in Chan's work [7], the proposed method of directly solving three simultaneous nonlinear equations is much easier. In this paper, the nonlinear simultaneous equations have been solved using Mathcad [13], which is a widely used general-purpose mathematic software tool.

\section{Numerical Applications}

An induction motor working as an SEIG supplying a single-phase load has been used to illustrate the proposed methods for solving $C_{2}$ and $C_{3}$ to balance the generator. It has been set up as shown in Figure 5. The generator is a 3-phase, $220-\mathrm{V}, \Delta$-connected, $60-\mathrm{Hz}$, 4-pole, $1 / 2 \mathrm{hp}$ squirrel-cage induction machine. The motor characteristics and test methods for obtaining the parameters of the generator equivalent circuit are given in the appendix. Also included in the appendix are the test method for the magnetizing saturation curve, and how the fixed capacitor $C_{1}$ is determined in this study.

Figure 5. Experimental set-up of an SEIG feeding a single-phase load.

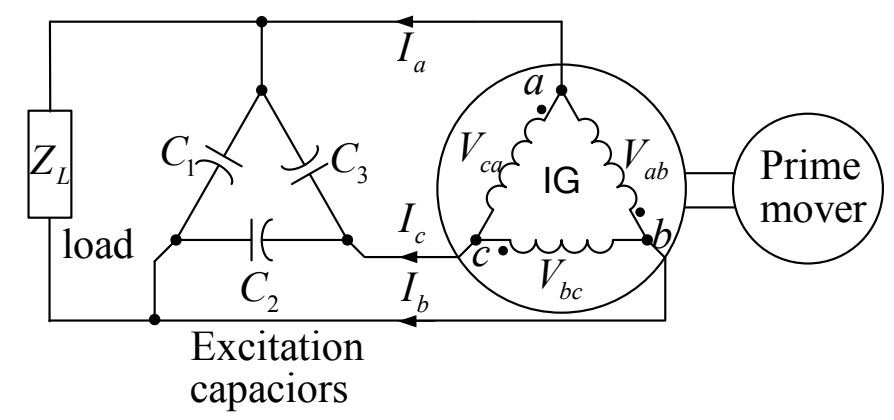

In the following sections, we firstly show a few examples of applying the proposed solution method for finding the values of $C_{2}$ and $C_{3}$ that balance the generator when $Z_{L}, C_{1}$ and the rotational speed are known. The example that follows is the use of the capacitance values found by the solution method for the experiment on the $1 / 2 \mathrm{hp}$ squirrel-cage induction machine to see whether the generator is working under balanced conditions.

\subsection{Verification of the Obtained Capacitance Values}

The solution method described in Section 3 has been employed to find the values of $C_{2}$ and $C_{3}$ when the generator runs at $1764 \mathrm{rpm}$ and $C_{1}$ is fixed to $10 \mu \mathrm{F}$. The solved results for resistive loads 
$Z_{L}=400 \Omega$ and $Z_{L}=1000 \Omega$, and inductive loads $Z_{L}=500+j 1130.97 \Omega$ and $Z_{L}=467.13+j 123.91 \Omega$ (at $60 \mathrm{~Hz}$ ) are shown in Table 1.

In the table, the values of $\tau$ corresponding to the solution values of $C_{2}$ and $C_{3}$ are also listed, being all very close to zero and showing that the obtained capacitance values can balance the generator. The values of the magnetizing reactance $X_{m}$ listed in Table 1 are all smaller than $X_{c r} / F$, meaning that the capacitance values obtained allow the generator to build voltage.

Table 1. Capacitance values $C_{2}$ and $C_{3}$ and the resulting voltage unbalance factor for different load impedances at a rotating speed of $n=1764 \mathrm{rpm}$.

\begin{tabular}{ccccc}
\hline $\boldsymbol{n}$ (rpm) & \multicolumn{4}{c}{$\mathbf{1 7 6 4}$} \\
\hline $\boldsymbol{Z}_{\boldsymbol{L}}(\Omega)$ & $\mathbf{4 0 0} \Omega$ & $\mathbf{1 0 0 0} \Omega$ & $\mathbf{R}-\mathbf{L}$ (series) & $\mathbf{R} / \mathbf{L}$ (parallel) \\
& & & $\mathbf{R}=\mathbf{5 0 0} \Omega, \mathbf{L}=\mathbf{3 0 0 0} \mathbf{~} \mathbf{H}$ & $\mathbf{R}=\mathbf{5 0 0} \Omega, \mathbf{L}=\mathbf{5 0 0 0} \mathbf{~ m H}$ \\
\hline$C_{2}(\mu \mathrm{F})$ & 13.98 & 11.58 & 8.490 & 11.66 \\
$C_{3}(\mu \mathrm{F})$ & 6.019 & 8.421 & 7.420 & 5.321 \\
$\tau(\%)$ & $1.86 \times 10^{-6}$ & $2.97 \times 10^{-7}$ & $1.62 \times 10^{-7}$ & $3.7 \times 10^{-7}$ \\
$P_{\text {out }}(W)$ & 125.76 & 57.27 & 9.17 & 59.20 \\
$F($ p.u. $)$ & 0.96182 & 0.97004 & 0.97543 & 0.96590 \\
$I_{m}(A)$ & 1.36 & 1.49 & 0.83 & 0.90 \\
$X_{m}(\Omega)$ & 93.44 & 89.10 & 110.98 & 108.98 \\
\hline
\end{tabular}

The values of $C_{2}$ and $C_{3}$ solved by the proposed method for operating conditions of $n=1800 \mathrm{rpm}$ with load $Z_{L}$ equal to $500,2000,600+j 1357.16$ and $373.7+j 99.12 \Omega$ (at $60 \mathrm{~Hz}$ ), respectively, are listed in Table 2, in which the resulting voltage unbalance factors $\tau$ are small too, verifying that the obtained capacitance values can balance the generator. Checks on the magnetizing reactance $X_{m}$ also assure building-up of the generator voltage.

Table 2. Values of $C_{2}$ and $C_{3}$ for different load impedances at a speed of $n=1800 \mathrm{rpm}$.

\begin{tabular}{|c|c|c|c|c|}
\hline$n(\mathbf{r p m})$ & & & 1800 & \\
\hline$Z_{L}(\Omega)$ & $500 \Omega$ & $2000 \Omega$ & $\begin{array}{c}\text { R-L (series) } \\
R=600 \Omega, L=3600 \mathrm{mH}\end{array}$ & $\begin{array}{c}R / / \mathrm{L}(\text { parallel }) \\
\mathrm{R}=\mathbf{4 0 0} \Omega, \mathrm{L}=\mathbf{4 0 0 0} \mathrm{mH}\end{array}$ \\
\hline$C_{2}(\mu \mathrm{F})$ & 13.11 & 10.77 & 8.769 & 12.07 \\
\hline$C_{3}(\mu \mathrm{F})$ & 6.887 & 9.229 & 7.930 & 4.285 \\
\hline$\tau(\%)$ & $3.18 \times 10^{-7}$ & $1.94 \times 10^{-7}$ & 0.0041 & 0.00019 \\
\hline$P_{\text {out }}(W)$ & 118.98 & 33.22 & 12.35 & 69.35 \\
\hline$F$ (p.u.) & 0.98395 & 0.99249 & 0.99528 & 0.98297 \\
\hline$I_{m}(A)$ & 1.53 & 1.66 & 1.14 & 0.85 \\
\hline$X_{m}(\Omega)$ & 88.09 & 84.13 & 100.93 & 110.46 \\
\hline
\end{tabular}

The values of $C_{2}$ and $C_{3}$ found for operating conditions of $n=1836 \mathrm{rpm}$ with load $Z_{L}$ equal to 600 , $3000,700+j 1507.96$ and $560.55+j 148.69 \Omega$ (at $60 \mathrm{~Hz}$ ), respectively, are summarized in Table 3 . The resulting voltage unbalance factors are again very close to zero. Voltage building-up is also checked for each loading condition. 
Table 3. Values of $C_{2}$ and $C_{3}$ for different load impedances at a speed of $n=1836 \mathrm{rpm}$.

\begin{tabular}{|c|c|c|c|c|}
\hline$n(\mathbf{r p m})$ & & & 1836 & \\
\hline$Z_{L}(\Omega)$ & $600 \Omega$ & $3000 \Omega$ & $\begin{array}{c}\text { R-L (series) } \\
R=700 \Omega, L=4000 \mathrm{mH}\end{array}$ & $\begin{array}{c}\mathrm{R} / / \mathrm{L} \text { (parallel) } \\
\mathbf{R}=\mathbf{6 0 0} \Omega, \mathrm{L}=\mathbf{6 0 0 0} \mathrm{mH}\end{array}$ \\
\hline$C_{2}(\mu \mathrm{F})$ & 12.54 & 10.50 & 8.961 & 11.38 \\
\hline$C_{3}(\mu \mathrm{F})$ & 7.461 & 9.496 & 8.215 & 6.306 \\
\hline$\tau(\%)$ & $1.12 \times 10^{-6}$ & $5.88 \times 10^{-9}$ & $2.25 \times 10^{-7}$ & $3.97 \times 10^{-8}$ \\
\hline$P_{\text {out }}(W)$ & 113.21 & 24.73 & 14.03 & 89.10 \\
\hline$F$ (p.u.) & 1.00527 & 1.01311 & 1.01499 & 1.00648 \\
\hline$I_{m}(A)$ & 1.67 & 1.79 & 1.35 & 1.31 \\
\hline$X_{m}(\Omega)$ & 83.59 & 80.28 & 93.93 & 95.05 \\
\hline
\end{tabular}

\subsection{Experimental Results}

The 1/2 hp squirrel-cage induction machine operating as an SEIG is set up as shown in Figure 5 for experiments. The SEIG's rotational speed and excitation capacitor $C_{1}$ are fixed at $1800 \mathrm{rpm}$ and $10 \mu \mathrm{F}$, respectively. Both pure resistive loads and inductive loads are tested.

For the case of pure resistive loads, when the load $Z_{L}$ varies from 500 to $2000 \Omega$, the proposed solution method is used to find the corresponding values of $C_{2}$ and $C_{3}$ that balance that generator. These capacitance values are then used in the two-port network model and in the experiments. The capacitance values obtained by the solution method are directly used as the inputs to the two-port network method. However, for the experiments, the (parallel and series combinations of) capacitors with values closest to the calculated values are used. Table 4 lists the load resistance values, the capacitance values calculated by solving Equations $(16,21,22)$, the capacitance values actually used in the experiments, the measured values of voltage unbalance factor (VUF) and the current unbalance factor (CUF). The measured VUF values listed in Table 4 are all lower than $1 \%$, validating the proposed method. Figure 6 compares the measured values (scattered dots) of frequency, three-phase voltages, currents and output power, with the results obtained by the proposed analytical method (solid lines), for the resistive load continuously varying from 500 to $2000 \Omega$. Good agreement has been achieved. The comparison of line voltages and currents depicted in Figures 6(b,c) only shows the values in one phase for the calculated results (solid lines) since the calculated voltages and currents are nearly perfectly balanced. Nevertheless, the measured three-phase voltages and currents are given in detail to allow the unbalance to be observed.

The experimental results for inductive loads are summarized in Table 5. Since the impedance value does not vary continuously, it is not appropriate to present these results graphically, so only a table is given. In this table, both calculated and actually used capacitance values are listed. The load resistance is easily adjusted using a rheostat, but the load inductance is more unstable because of magnetic saturation. The experiment has been carried out by firstly choosing an approximate value of inductance, and then finding its accurate inductance value by calculation from the measured voltage and current of the inductor. After the load resistance and inductance are obtained, the same procedure as in the case of pure resistive loads has been followed to find the capacitances $C_{2}$ and $C_{3}$. Also listed in Table 5 are measured VUF and CUF values. Except for the first row, the VUF values are all lower than 1\%. The 
VUF value in the first row is higher because the value of $C_{3}$ used in the experiment deviates slightly more significantly from its theoretical value than those in other rows.

Figure 6. Comparison of the results obtained by experiments (scattered dots) and by the analytical method (solid line) for resistive load $Z_{\mathrm{L}}$ varying from $500 \Omega$ to $2000 \Omega$. (a) Frequency; (b) Line voltages; (c) Line currents; (d) Output power.

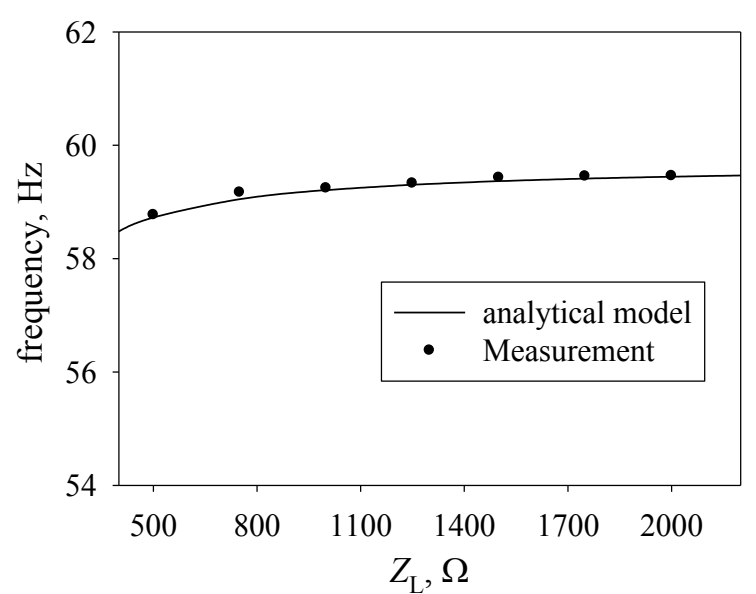

(a)

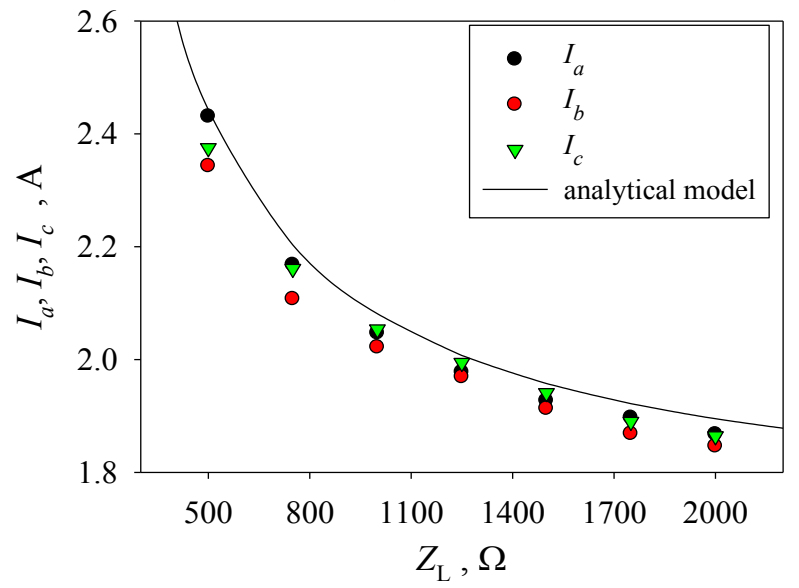

(c)

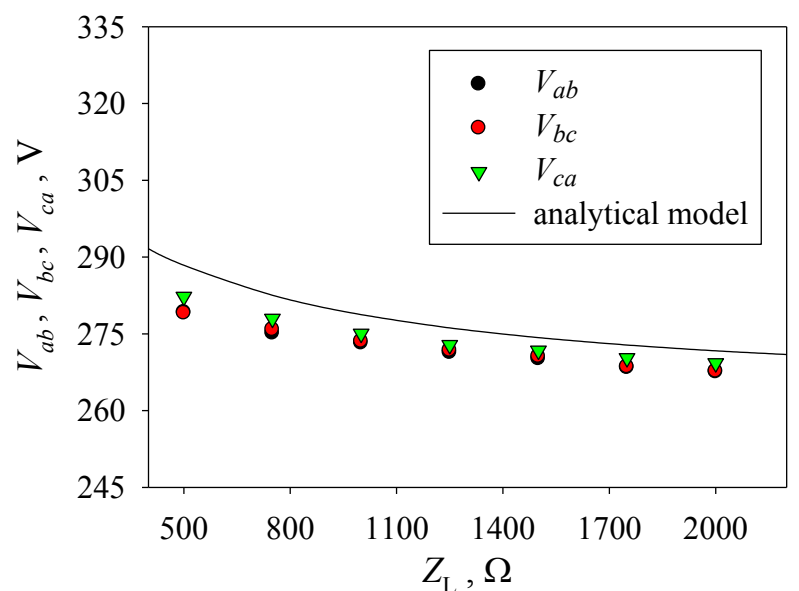

(b)

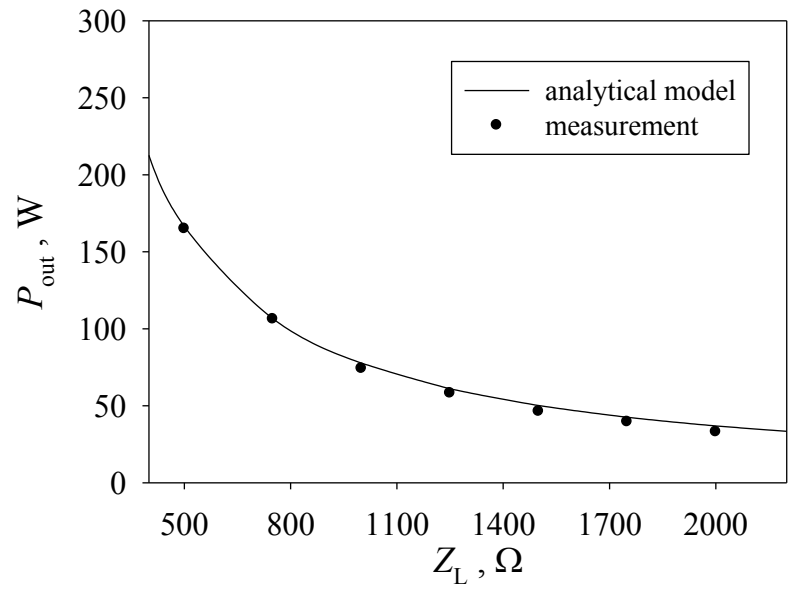

(d)

Table 4. Theoretical and experimental capacitance values and measured VUF and CUF for pure resistive loads.

\begin{tabular}{ccccccc}
\hline $\boldsymbol{Z}_{\mathbf{L}}(\boldsymbol{\Omega})$ & \multicolumn{2}{c}{ Theoretical } & \multicolumn{4}{c}{ Experimental } \\
\cline { 2 - 6 } (resistive) & $\boldsymbol{C}_{\mathbf{2}}(\boldsymbol{\mu} \mathbf{F})$ & $\boldsymbol{C}_{\mathbf{3}}(\boldsymbol{\mu} \mathbf{F})$ & $\boldsymbol{C}_{\mathbf{2}}(\boldsymbol{\mu} \mathbf{F})$ & $\boldsymbol{C}_{\mathbf{3}}(\boldsymbol{\mu} \mathbf{F})$ & VUF $(\%)$ & CUF $(\%)$ \\
\hline 500 & 13.11 & 6.888 & 13.2 & 6.8 & 0.74 & 1.96 \\
750 & 12.07 & 7.933 & 12.1 & 8.0 & 0.60 & 1.70 \\
1,000 & 11.55 & 8.452 & 11.5 & 8.5 & 0.40 & 0.82 \\
1,250 & 11.24 & 8.763 & 11.2 & 8.7 & 0.31 & 0.62 \\
1,500 & 11.03 & 8.970 & 11.0 & 9.0 & 0.35 & 0.71 \\
1,750 & 10.88 & 9.118 & 10.8 & 9.1 & 0.44 & 0.86 \\
2,000 & 10.77 & 9.228 & 10.6 & 9.2 & 0.40 & 0.83 \\
\hline
\end{tabular}


Table 5. Theoretical and experimental capacitance values and measured VUF and CUF for inductive loads.

\begin{tabular}{cccccccc}
\hline \multicolumn{2}{c}{$\boldsymbol{Z}_{\mathbf{L}}=\boldsymbol{R}+\boldsymbol{j} \boldsymbol{\omega} \boldsymbol{L}$} & \multicolumn{2}{c}{ Theoretical } & \multicolumn{3}{c}{ Experimental } \\
\hline $\boldsymbol{R}(\boldsymbol{\Omega})$ & $\boldsymbol{L}(\mathbf{m H})$ & $\boldsymbol{C}_{\mathbf{2}}(\boldsymbol{\mu} \mathbf{F})$ & $\boldsymbol{C}_{\mathbf{3}}(\boldsymbol{\mu} \mathbf{F})$ & $\boldsymbol{C}_{\mathbf{2}}(\boldsymbol{\mu} \mathbf{F})$ & $\boldsymbol{C}_{\mathbf{3}}(\boldsymbol{\mu} \mathbf{F})$ & VUF $(\%)$ & $\mathbf{C U F}(\%)$ \\
\hline 50 & 1663.56 & 14.47 & 10.40 & 14.45 & 10.24 & 1.02 & 5.54 \\
150 & 1701.34 & 14.49 & 11.07 & 14.60 & 11.07 & 0.28 & 0.84 \\
400 & 1787.83 & 13.95 & 12.02 & 14.10 & 11.95 & 0.51 & 0.59 \\
600 & 1888.30 & 13.27 & 12.14 & 13.35 & 12.10 & 0.21 & 0.46 \\
\hline
\end{tabular}

\section{Conclusions}

This paper has proposed a three-capacitor circuit scheme that is able to balance an SEIG supplying a single-phase load. The three-capacitor scheme consists of a fixed capacitor and two variable capacitors. The fixed capacitor is chosen to compensate the reactive power required by the generator and the load, while the two variable capacitors serve to balance the generator according to the load impedance and the rotational speed of the generator.

In order to find the values of the two variable capacitors, a two-port network model of the SEIG has been employed to analyze the generator with arbitrary unbalanced loads. With the aid of this circuit model, a method that involves solving a set of three nonlinear simultaneous equations has been proposed to find the values of the two capacitors that minimize the voltage unbalance of the generator. The proposed method has further been validated by experiments on a $1 / 2 \mathrm{hp}$ three-phase squirrel-cage induction motor working as an induction generator feeding a single-phase load. Both resistive and inductive single-phase loads have been tested and satisfactory results have been obtained.

\section{Acknowledgments}

This research was financially supported by the National Science Council of Taiwan, ROC, under grant number NSC101-2221-E-224-074.

\section{Appendix}

\section{A.1. Generator Characteristics, Test Methods and Saturation Curve}

The generator used is a squirrel-cage induction motor working as an SEIG. The motor is 3-phase, $220-\mathrm{V}, \Delta$-connected, $60-\mathrm{Hz}, 4-$ pole, and rated at $1 / 2 \mathrm{hp}$. The no-load test, the blocked-rotor test and the DC test for stator resistance were carried out to obtain the parameters of the motor equivalent circuit. The procedures of these tests are described in detail in most textbooks of electrical machines such as [14]. The equivalent circuit parameters are $R_{s}=20.63 \Omega, R_{r}=15.85 \Omega, X_{S}=X_{r}=21.062 \Omega$.

Another test for tracing the magnetizing saturation curve was also performed at $60 \mathrm{~Hz}(F=1)$. The motor shaft was coupled to a synchronous motor to keep its speed at $1800 \mathrm{rpm}$. The three-phase motor terminal voltage was varied from nearly zero to a value that slightly overcurrented the motor. The terminal voltage subtracted by the voltage drop of the stator impedance allows the air gap voltage $V_{g}$ to be found. The curve of the air gap voltage $V_{g}$ against the measured stator current, which is in this 
condition equal to the magnetizing current $I_{m}$, is the magnetizing saturation curve as shown in Figure A1. Figure A1 shows the measured results (scattered dots) and a curve with the expression:

$$
\frac{V_{g}}{F}=\frac{a}{1+\left(I_{m} / b\right)^{-c}}
$$

where parameters $a=183.3082 \mathrm{~V}, b=0.8697 \mathrm{~A}$ and $c=1.5704$ were found using a nonlinear regression tool. Figure A1 allows the value of the critical magnetizing reactance $X_{c r}$ to be obtained by drawing a straight line passing through the origin and tangent to the magnetizing curve. The slope of this line yields a good estimate of $X_{c r}$. The value $X_{c r}=113 \Omega$ at $60 \mathrm{~Hz}$ (i.e., $F=1.0$ ) is used in the analysis. It is noted that this value needs to be corrected by the per-unit frequency $F$ for a frequency other than $60 \mathrm{~Hz}$.

Figure A1. Magnetizing curve of the SEIG.

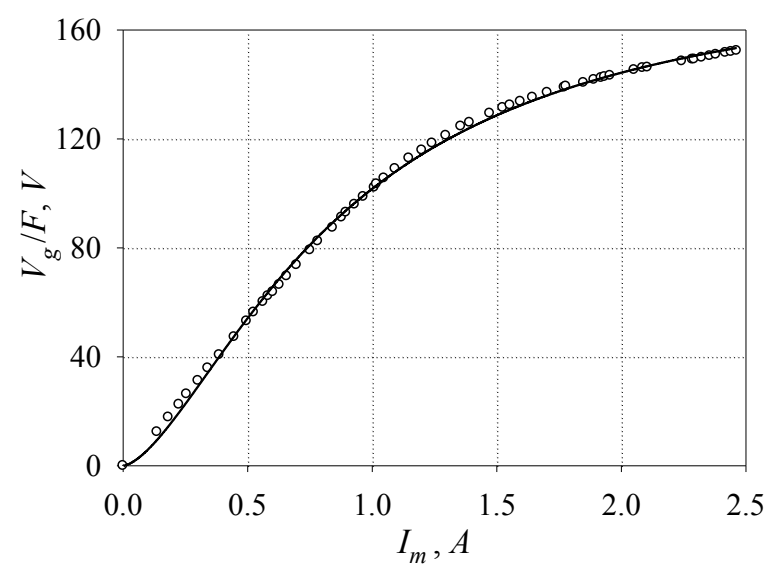

\section{A.2. Determination of Capacitance $\mathbf{C}_{1}$}

Capacitance $C_{1}$ is set to a fixed value of $10 \mu \mathrm{F}$ throughout the study. This value was obtained in the balanced loading test of the generator. When the excitation capacitors are all equal to $10 \mu \mathrm{F}$, i.e., $C_{1}=C_{2}=C_{3}=10 \mu \mathrm{F}$, and the three-phase load is also balanced, the generator easily builds up voltage over a wide range of load resistance. The value of $10 \mu \mathrm{F}$ is thus taken as a fixed value for $C_{1}$ which is not variable in this study. In our tests, a higher value of $C_{1}$ also allows the generator to build up voltage easily, but the generator also attains an overcurrent condition. On the other hand, a lower value of $C_{1}$ sometimes fails to build up voltage. Hence, the value of $10 \mu \mathrm{F}$ is a compromise of voltage building up and the generator current rating.

\section{A.3. Physical Implementation of Variable Capacitors}

The two variable capacitors $C_{2}$ and $C_{3}$ in Figure 1 serve to balance the generator. In electric grid applications, an old method of adjusting capacitance is the use of a no-load synchronous motor. Its excitation current is controlled to generate variable reactive power, and the synchronous motor acts as a variable capacitor. Modern power electronics technology has allowed this old method to be replaced by SVC which is composed of a TCR with a shunt capacitor, and has been extensively employed in the electric grid for reactive power control. The basic principle of SVC is illustrated in Figure A2. If a 
variable capacitor across nodes $a$ and $b$ is needed as shown in Figure A2a, then a parallel combination of a fixed capacitor $C_{S}$ and a variable inductor $L$ (see Figure $\mathrm{A} 2 \mathrm{~b}$ ) has the equivalent function as a variable capacitor. The variable inductor $L$ can be realized by a TCR which is basically a fixed inductor $L_{T}$ controlled by two back-to-back thyristors as shown in Figure A2c. By controlling firing angle $\alpha$ of the thyristors, the current flowing through inductor $L_{T}$ can be adjusted continuously from a maximum value at full conduction to a minimum value (zero) at cut-off of the thyristors. Figure A3 shows a possible application of two SVCs for the implementation of variable capacitors $C_{2}$ and $C_{3}$ shown in Figure 1. By controlling firing angles $\alpha_{1}$ and $\alpha_{2}$, the capacitance values of $C_{3}$ and $C_{2}$ can be varied, respectively.

Figure A2. Basic principle of realizing a variable capacitor using an SVC. (a) A variable capacitor $C$; (b) A fixed capacitor $C_{S}$ in parallel with a variable inductor $L$; (c) An SVC composed of a TCR with a shunt capacitor $C_{S}$.

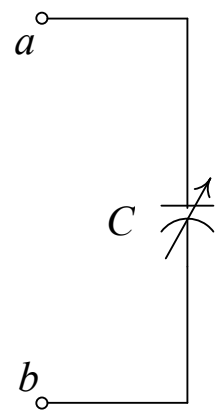

(a)

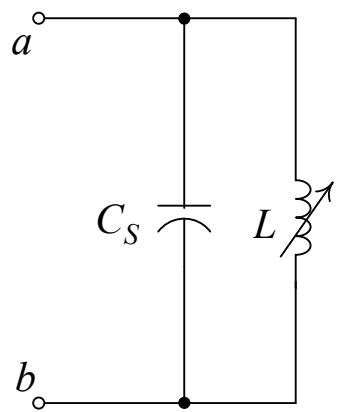

(b)

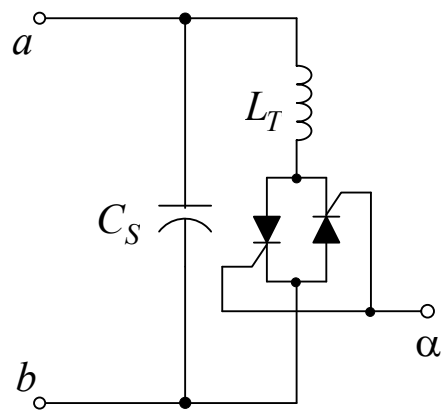

(c)

Figure A3. Implementation of variable capacitors $C_{2}$ and $C_{3}$ using two SVCs.

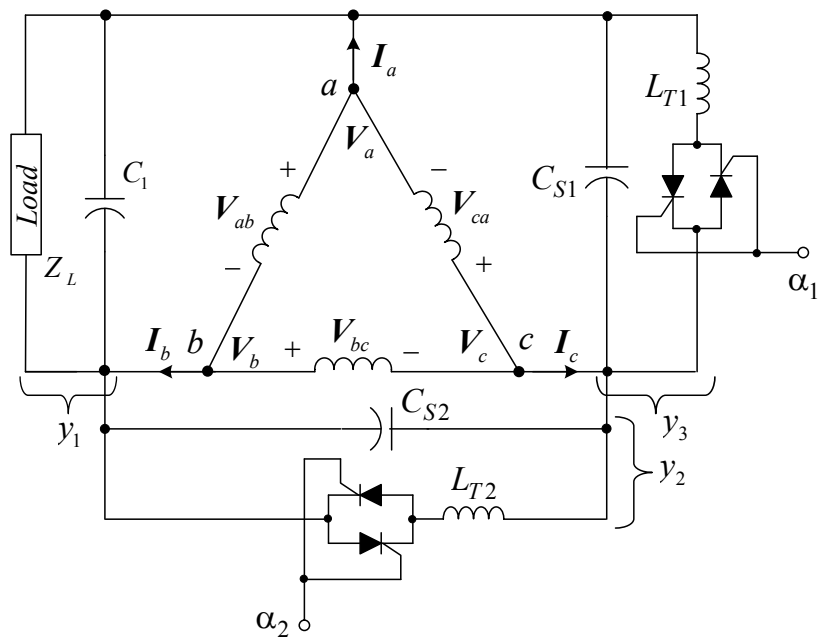

\section{References}

1. Murthy, S.S.; Singh, B.; Gupta S.; Gulati, B.M. General steady-state analysis of three-phase self-excited induction generator feeding three-phase unbalanced load/single-phase load for stand-alone applications. IEE Proc. Gener. Transm. Distrib. 2003, 150, 49-55. 
2. Durham, M.O.; Ramakumar, R. Power system balancers for an induction generator. IEEE Trans. Ind. Appl. 1987, 23, 1067-1072.

3. Wang, L.; Cheng, C.M. Excitation capacitance required for an isolated three-phase induction generator under single-phasing mode of operation. In Proceedings of International Conference of Power Engineering Society Winter Meeting, Columbus, OH, USA, 27-31 January 2001.

4. Fukami, T.; Kaburaki, Y.; Kawahara, S.; Miyamoto, T. Performance analysis of a self-regulated self-excited single-phase induction generator using a three-phase machine. IEEE Trans. Energy Convers. 1999, 14, 622-627.

5. Mahto, S.N.; Singh, S.P.; Sharma, M.P. Capacitors required for maximum power of a self-excited single-phase induction generator using a three-phase machine. IEEE Trans. Energy Convers. 2008, 23, 372-381.

6. Al-Bahrani, A.H.; Malik, N.H. Steady-state analysis and performance characteristic of a 3-phase induction generator self-excited with a single capacitor. IEEE Trans. Energy Convers. 1990, 5, $725-732$.

7. Chan, T.F. Performance analysis of a three-phase induction generator self-excited with a single capacitance. IEEE Trans. Energy Convers. 1999, 14, 894-900.

8. Chan, T.F.; Lai, L.L. Capacitance requirements of a three-phase induction generator self-excited with a single capacitance and supplying a single-phase load. IEEE Trans. Energy Convers. 2002, 17, 90-94.

9. Chan, T.F.; Lai, L.L. Comments to the paper "Capacitance requirements of a three-phase induction generator self-excited with a single capacitance and supplying a single-phase load". IEEE Trans. Energy Convers. 2004, 19, 222.

10. Chan, T.F.; Lai, L.L. Phase balancing for a self-excited induction generator. In Proceedings of International Conference of Power Utility Deregulation, Restructuring and Power Technologies, London, UK, 4-7 April 2000.

11. Alolah, A.I.; Alkanhal, M.A. Excitation requirements of three-phase self-excited induction generator under single-phase loading with minimum unbalance. In Proceedings of International Conference of Power Engineering Society Winter Meeting, Singapore, 23-27 January 2000.

12. Wang, Y.J.; Huang, S.Y. Analysis of a stand-alone three-phase self-excited induction generator with unbalanced load using a two-port network model. IET Electr. Power Appl. 2009, 3, 445-452.

13. Ansari, K.A. An Introduction to Numerical Methods Using Mathcad; Schroff Development Corp.: Spokane, WA, USA, 2007.

14. Chapman, S.J. Electric Machinery Fundamentals, 4th ed.; McGraw-Hill: Boston, MA, USA, 2005; pp. 452-460.

(C) 2012 by the authors; licensee MDPI, Basel, Switzerland. This article is an open access article distributed under the terms and conditions of the Creative Commons Attribution license (http://creativecommons.org/licenses/by/3.0/). 